\title{
Neuronal and Axonal Loss Are Selectively Linked to Fibrillar Amyloid- $\beta$ within Plaques of the Aged Primate Cerebral Cortex
}

\author{
Palak Shah, ${ }^{*}$ Neeta Lal, ${ }^{*}$ Elaine Leung, ${ }^{*}$ \\ David E. Traul, ${ }^{*}$ Alicia Gonzalo-Ruiz, ${ }^{\dagger}$ \\ and Changiz Geula* \\ From the Laboratory for Cognitive and Molecular Morphometry,* \\ the Cognitive Neurology and Alzheimer's Disease Center, \\ Northwestern University, Feinberg School of Medicine, Chicago, \\ Illinois; and the Laboratory of Neuroanatomy, ${ }^{\dagger}$ the Institute of \\ Neuroscience of Castilla and Leon, University of Valladolid, \\ Soria, Spain
}

The amyloid- $\beta$ peptide (A $\beta$ ) deposited in plaques in Alzheimer's disease has been shown to cause degeneration of neurons in experimental paradigms in vivo and in vitro. However, it has been difficult to convincingly demonstrate toxicity of native amyloid deposits in the aged and Alzheimer brains. Here we provide evidence that the fibrillar conformation of $\mathrm{A} \beta$ (fA $\beta$ ) deposited in compact plaques is associated with the pathologies observed in Alzheimer brains. fA $\beta$ containing compact but not diffuse plaques in the aged rhesus cortex contained activated microglia and clusters of phosphorylated tau-positive swollen neurites. Scholl's quantitative analysis revealed that the area adjacent to $\mathrm{fA} \beta$, containing compact but not diffuse plaques in aged rhesus, aged human, and Alzheimer's disease cortex, displays significant loss of neurons and small but statistically significant reduction in the density of cholinergic axons. These observations suggest that fA $\beta$ toxicity may not be restricted to cultured cells and animal injection models. Rather, fA $\beta$ deposited in native compact plaques in aged and $\mathrm{AD}$ brains may exert selective toxic effects on its surrounding neural environment. (Am J Pathol 2010, 177:325-333; DOI: 10.2353/ajpath.2010.090937)

A large body of evidence supports a central role for the amyloid- $\beta$ peptide $(A \beta)$ in the pathogenesis of Alzheimer's disease (AD). ${ }^{1}$ Deposition of $A \beta$ in plaques represents a signature pathological hallmark of $A D$. $A \beta$ can exist in various physical conformations, including soluble oligomers (oA $\beta$ ), protofibrils ( $\mathrm{pf} A \beta)$, nonfibrillar insoluble oligomers, and fibrillar ( $\mathrm{f} A \beta$ ) forms. ${ }^{2-6} A \beta$ immunoreactive plaques are of two primary types: diffuse and compact. $^{7,8}$ Diffuse plaques contain primarily nonfibrillar insoluble oligomers of $A \beta$. Compact plaques, on the other hand, are composed of $\mathrm{fA} \beta$ and have consistently been found to contain significantly larger numbers of activated microglia than adjacent brain regions and to be surrounded by astrocytes. ${ }^{9-13}$ They also contain abnormal neurites (neuritic plaques), which represent dystrophic processes of neurons and are composed of abnormally phosphorylated tau. ${ }^{9,14}$ Importantly, the presence of the compact neuritic variety of plaques that contain $\mathrm{f} A \beta$ and activated microglia appears to be a relatively specific feature of $A D$, and its density is used for the pathological diagnosis of the disease. ${ }^{9,15}$ Moreover, a strong correlation has been reported between the density of neuritic plaques and severity of dementia. ${ }^{16,17}$

The neuritic pathology associated with $\mathrm{f} A \beta$ is suggestive of the toxic effects of this conformation of the peptide on neurons and their processes. In fact, in vitro and in vivo evidence indicates that $\mathrm{f} A \beta$ exerts powerful toxic effects on neurons. ${ }^{18-20}$ We have shown that the aged primate cerebral cortex is selectively vulnerable to $\mathrm{f} A \beta$ toxicity. ${ }^{21}$ Injections of plaque equivalent concentrations (200 pg) of $\mathrm{fA} \beta$ into the cerebral cortex of aged rhesus or mormoset monkeys produced significant neuronal loss and induced hyperphosphorylation of tau, both features of the $\mathrm{AD}$ brain. Recent in vitro evidence indicates that $\mathrm{OA} \beta$, which are believed to form before deposition of $A \beta$ in plaques and are likely to interfere with synaptic function $^{22,23}$ and inhibit fast axonal transport, ${ }^{24}$ may also lead to neuronal degeneration. ${ }^{25-28}$ However, although there is abundant evidence demonstrating that oligomeric $A \beta$

Supported in part by the Alzheimer's Association (IIRG-2366) and by the Dana Foundation Neuroimmunology Program.

Accepted for publication March 12, 2010.

Address reprint requests to Changiz Geula, Ph.D., Laboratory for Cognitive and Molecular Morphometry, Cognitive Neurology and Alzheimer's Disease Center, Northwestern University, Feinberg School of Medicine 320 East Superior Street, Searle 11-467A, Chicago, IL 60611. E-mail: c-geula@northwestern.edu. 
interferes with neuronal function, direct in vivo demonstration that this conformation of $A \beta$ causes neuronal death is lacking. An exception is the demonstration of synaptic degeneration associated with intracellular accumulation of $A \beta$, most likely of the soluble oligomeric variety. ${ }^{29,30}$

The experimentally observed toxic effects of injected $A \beta$ imply that deposition of this peptide in plaques should also be associated with neuronal damage. However, it has been difficult to convincingly demonstrate neuronal and axonal loss associated with plaques in aged human or AD brains. A number of isolated studies have reported on limited aspects of neuronal and axonal damage in plaques, ${ }^{31-33}$ sometimes using very small numbers of specimens. ${ }^{34}$ Furthermore, none of these studies has addressed the potential differences in $\mathrm{fA} \beta$ and large oligomeric $A \beta$ in inducing toxic effects in plaques. Recently, neuronal loss has been demonstrated within the region occupied by $\mathrm{fA} \beta$ in plaques. ${ }^{35}$ However, this phenomenon could be attributed to physical damage to neurons by the space occupying amyloid. If $A \beta$ exerts toxic effects on neurons, neuronal and axonal loss should be observable in the area surrounding the plaque.

In the present set of experiments, we provide evidence suggesting that $\mathrm{f} A \beta$ exerts toxic effects on neurons and axons in the immediate area next to plaques. We first used plaques in the aged rhesus cortex for this purpose, because such plaques exist in an otherwise intact cortical architecture and only a fraction of them contain $\mathrm{fA} \beta$. We then extended our observations to normal human brains and AD cases. We report activated microglia, and for the first time the existence of phosphorylated tau in swollen neurites, exclusively associated with $f A \beta$ in plaques in the aged rhesus cortex. We also demonstrate significant loss of neurons and of cholinergic axons, which are selectively vulnerable to degeneration in $A D,{ }^{36}$ in the immediate vicinity of compact plaques containing $\mathrm{fA} \beta$ but not next to diffuse plaques. The loss of neurons and axons becomes progressively smaller with distance away from such plaques. Additionally, we demonstrate that $f A \beta$ containing compact plaques in the aged human and $A D$ brains display significant neuronal loss in their immediate vicinity.

\section{Materials and Methods}

\section{Cases and Tissue Processing}

Eleven aged (25 to 31 years old, 3 males and 8 females) specific pathogen-free rhesus monkeys, with no neurological disorders or other injuries that can cause trauma to the central nervous system, were obtained from Charles River Primate (Summerland, FL) and used in this study. The birth date of each animal was known so that the exact age of each could be determined with certainty.

Three animals received an overdose of anesthetic (12 $\mathrm{mg} / \mathrm{kg}$ ketamine followed by $100 \mathrm{mg} / \mathrm{kg}$ sodium pentobarbital) and were perfused intracardially with saline (500 $\mathrm{ml}$ ) followed by $4 \%$ paraformaldehyde in $0.1 \mathrm{M}$ phosphate buffer ( $\mathrm{pH} 7.4 ; 1.5$ to 2 liters) and $10 \%$ sucrose in $0.1 \mathrm{M}$ phosphate buffer. Then the brains were removed and taken through additional sucrose gradients (20 to $30 \%)$ for cryoprotection. The remaining eight animals received an overdose of anesthetic and the brains were removed, blocked, and placed in $4 \%$ paraformaldehyde for 24 hours at $4^{\circ} \mathrm{C}$ and taken through sucrose gradients.

For comparison with the rhesus, brains of two normal control (72-year-old male and 83-year-old female) and three clinically and pathologically confirmed AD (69-yearold male, 75-year-old male, and 89-year-old male) cases were used. These brains were blocked, fixed in $4 \%$ paraformaldehyde for 30 to 36 hours at $4^{\circ} \mathrm{C}$, and taken through sucrose gradients.

Each brain was sectioned serially at $40 \mu \mathrm{m}$ on a freezing microtome and stored in $0.1 \mathrm{M}$ phosphate buffer containing $0.02 \%$ sodium azide at $4^{\circ} \mathrm{C}$ until used.

\section{Immunohistochemistry}

Immunohistochemistry was performed according to the avidin-biotin-peroxidase complex (ABC) method using the Vectastain Elite Kit (Vector Laboratories, Burlingame, CA) as previously described. ${ }^{37}$ The following specific antibodies were used for this purpose: polyclonal antibody 1282 against $A \beta(1 / 2000$, gift of Dr. Dennis Selkoe, Harvard Medical School, Boston, MA); polyclonal antibody $B 7$ against $A \beta(1 / 2000$, gift of Dr. Bruce Yankner, Harvard Medical School); monoclonal antibody PHF1 which recognizes tau phosphorylated at Ser 396/404 (1/ 1000, gift of Dr. Peter Davies, Albert Einstein School of Medicine, New York, NY); monoclonal antibody to class II major histocompatibility glycoprotein HLA-DR (1/500; Dako, Glostrup, Denmark), a marker of microglia activation; and monoclonal antibody to microglia marker CD68 (1/500; Dako). Sections processed in the presence of irrelevant $\lg G$ instead of antibody or in the absence of primary antibody were used as controls.

\section{Cholinesterase Histochemistry}

We have previously shown that in the primate brain, acetylcholinesterase (AChE) activity specifically visualizes cortical cholinergic axons. ${ }^{38,39}$ Therefore, we used AChE histochemistry for determination of the status of these axons. Cortical cholinergic axons were visualized with the help of a highly sensitive AChE histochemical method. The principles of this method (incubation in a dilute Karnovsky-Roots medium followed by metal ion diaminobenzidine intensification) have been described by Hanker et $\mathrm{al}^{40}$ and Tago et al. ${ }^{41}$ We have introduced a number of changes in this method as described elsewhere. ${ }^{42}$ The specific inhibitor Iso-OMPA (Sigma Chemical Company, St. Louis, MO) was used to inhibit butyrylcholinesterase activity. The specific AChE inhibitor BW284C51 (Sigma Chemical Company) was used to ascertain specific AChE activity.

\section{Double Staining}

For concurrent visualization of two different antigens in the same tissue section, the double-immunohistochemi- 
cal method of Levy et $\mathrm{al}^{43}$ was used. For this purpose, tissue sections were first processed for the visualization of immunoreactivity of one antigen using diaminobenzidine (DAB) as chromogen. After the development of the $\mathrm{DAB}$ brown reaction product, the tissue sections were processed for visualization of the second antigen, except the peroxidase labeling was visualized using benzidine dihydrochloride (Sigma Chemical Company), which results in a granular blue reaction product.

For visualization of $\mathrm{f} A \beta$ in immunostained tissue, sections were first processed immunohistochemically and then were stained with thioflavin-S. Similarly, for visualization of cholinergic axons in the vicinity of plaques, sections were first stained immunohistochemically for visualization of $A \beta$ and then processed histochemically for staining AChE-positive cortical cholinergic axons. For the investigation of neuronal loss in the vicinity of plaques, sections processed immunohistochemically for visualization of $A \beta$ were counterstained for Nissl using the Cresyl violet stain. Double staining for Nissl using Cresyl violet and for $\mathrm{fA} \beta$ using thioflavin-S was not possible, as both stains are water soluble and processing for the second stain eliminates the first. Therefore, to ascertain specificity of neuronal loss in compact plaques containing $\mathrm{f} A \beta$, sections were first stained immunohistochemically for markers of activated microglia, which are exclusively present in thioflavin-S positive compact plaques that contain $\mathrm{fA} \beta$ (see Results), and then were processed for Nissl using the Cresyl violet stain.

\section{Quantitative Analysis}

For determination of the numbers of thioflavin-S-positive and thioflavin-S-negative plaques that contain activated microglia and phosphorylated tau-positive swollen neurites, plaques containing each element were counted in each double-stained section and expressed as the percentage of total fA $\beta$-positive thioflavin-S-stained plaques. Serial sections spanning the entire brain were used in this analysis.

To determine the extent of neuronal and cholinergic axonal loss in the vicinity of compact plaques, a modified Scholl's analysis was performed. For determination of neuronal loss, electronic photomicrographs of compact plaques containing $A \beta$ or activated microglia counterstained for Nissl were used. The plaque was placed inside a circle. Five concentric circles $20 \mu \mathrm{m}$ apart were drawn around this central circle. Then, the number of Nissl-stained neurons in each of five rings of tissue around the plaque defined by the circles was determined and expressed as number of neurons per $\mathrm{mm}^{2}$. The presence of large nuclei with prominent nucleoli was used to distinguish neurons from glial cells, which possess small nuclei without prominent nucleoli. The density of cholinergic axons around plaques was determined in a similar manner, except the intersection of axons with each of the five circles around plaques was determined and expressed per $\mathrm{mm}^{2}$.

As many as 20 representative sections of each brain were used in the quantitative analysis, and for the Scholl's analysis as many as 55 compact plaques and 46 diffuse plaques were chosen randomly for quantitation per case.

A test of normality demonstrated that all data were normally distributed. Therefore, analysis of variance with the Newman-Keuls post hoc test was used for determination of significant effects.

\section{Results}

\section{A $\beta$ Immunoreactive Plaques in the Aged Rhesus Cortex}

Consistent with our earlier report, ${ }^{44}$ we observed $A \beta$ immunoreactive deposits in the cerebral cortex of all aged rhesus ( 25 to 30 years) used in these experiments. As we had observed previously, ${ }^{44}$ the density of plaques was variable across animals. The cortex of some animals displayed a low density of plaques, while the cortex of others displayed a very high density. Two primary types of $A \beta$ deposits were observed. Diffuse $A \beta$ deposits were round or amorphous, stained relatively lightly, and lacked clear borders (Figure 1B). Compact $A \beta$ deposits were intensely stained round deposits with clear borders (Figure 1A). The overwhelming majority of plaques in the aged rhesus cortex were of the diffuse variety. A smaller

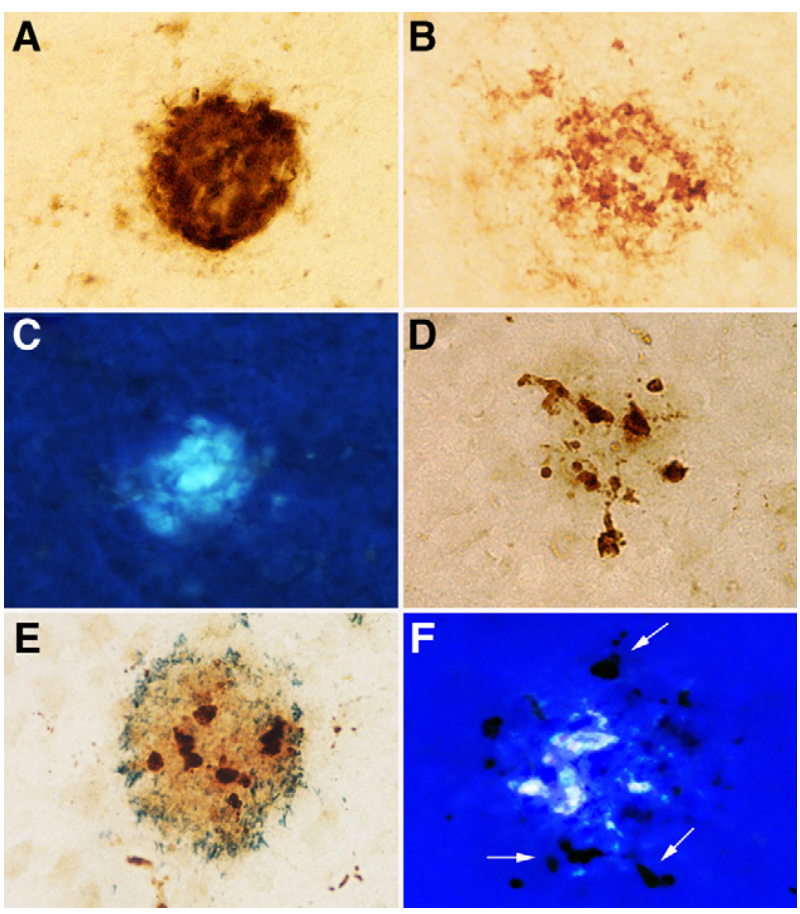

Figure 1. Microglia are colocalized with fibrillar amyloid- $\beta$ (fA $\beta$ ) in compact plaques of the aged rhesus cortex. A: Compact plaques in the aged rhesus cortex contained intense $\mathrm{A} \beta$ immunoreactivity and displayed well-defined borders. B: Diffuse plaques were lightly stained for $\mathrm{A} \beta$ and lacked clear borders. C: Thioflavin-S epifluorescence was present in compact plaques, indicative of the presence of $\mathrm{fA} \beta$. D: Immunoreactivity for HLA-DR, a marker of activated microglia, visualized clusters of microglia in plaque-like arrangements in the aged rhesus cortex. E: Double-staining for $\mathrm{A} \beta$ (blue) and the microglia marker CD68 (brown) indicated that nearly all such microglia clusters were within plaques. F: Double-staining for HLA-DR and thioflavin-S demonstrated that virtually all activated microglia clusters (arrows) in the aged rhesus cortex are within compact plaques containing fA $\beta$. Magnification in $\mathbf{A}-\mathbf{F}, \times 400$. 
number were morphologically of the compact variety. Consistent with this observation, a subpopulation of plaques was thioflavin-S (TS)-positive (Figure 1C). TS binds the $\beta$-pleated sheet conformation of $\mathrm{fA} \beta$ and thus is indicative of the presence of $\mathrm{f} A \beta$ within compact plaques. All brains contained both diffuse and compact plaques. We have estimated that approximately $21 \%$ of plaques in the aged rhesus cortex are compact and stain with TS. ${ }^{44}$

\section{Compact (TS-Positive) Rhesus Plaques Contain Activated Microglia and Swollen Neurites}

Staining for the microglia markers CD68 or the class II major histocompatibility glycoprotein (HLA-DR) revealed clusters of activated microglia in the cerebral cortex of aged rhesus monkeys (Figure 1D). Clusters were defined as two or more activated microglia in a plaque-like circular arrangement. Concurrent staining for the above markers and $A \beta$ indicated that activated microglia clusters are associated with $A \beta$, the major constituent of plaques (Figure 1E). Isolated single activated microglia were observed very rarely and were never associated with plaques. TS double staining showed that nearly all microglia clusters are found within $f A \beta$ containing compact plaques (Figure 1F). Quantitative analysis showed that 90 to $98 \%$ (percentage calculated for each animal) of microglia clusters are in TS-positive plaques and 83 to $90 \%$ of TS-positive plaques contain microglia clusters. Thus, virtually all microglia clusters are in plaques containing $\mathrm{f} A \beta$.

Immunoreactivity for the PHF1 epitope of abnormally phosphorylated tau was present within clusters of swollen neurites (Figure 2A), reminiscent of immature dystrophic neurites observed in plaques within $A D$ brains. Clusters were defined as two or more distinct swollen neurites in a plaque-like circular arrangement. Consistent with their
A

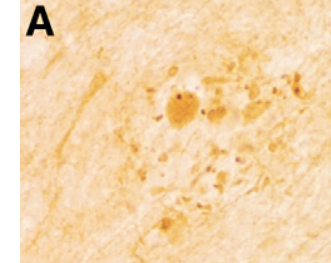

C

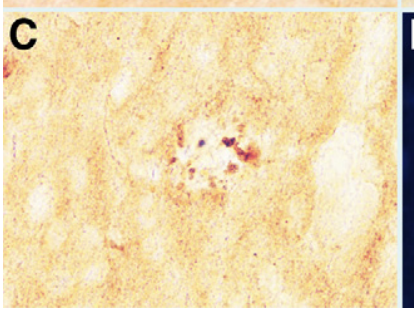

B
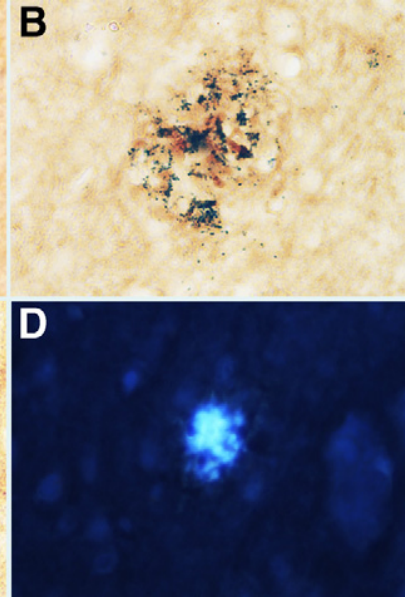

Figure 2. Abnormally phosphorylated tau (PHF-1) immunoreactivity in swollen neurites is present within fA $\beta$-containing compact plaques in the aged rhesus cortex. A: Clusters of PHF-1 immunoreactive swollen neurites were present in plaque-like arrangements in the aged rhesus cortex. B: Double staining for $\mathrm{A} \beta$ and PHF-1 demonstrated that nearly all such swollen neurites (brown) were located within $\mathrm{A} \beta$ deposits. (blue) $\mathbf{C}$ : Light microscopy in PHF-1 and thioflavin-S double stained material demonstrated the presence of abnormal swollen neurites in plaque-like arrangements. D: Presence of thioflavin-S epifluorescence in the same section demonstrated that virtually all PHF-1 swollen neurites were within compact plaques containing fA $\beta$. Magnification in $\mathbf{A}-\mathbf{D}, \times 400$. immature appearance, clusters of swollen neurites in the aged rhesus cortex were not TS-positive. Swollen neurites were present in the cerebral cortex of every aged rhesus brain examined. Concurrent staining for PHF1 and $A \beta$ showed that virtually all (up to $100 \%$ ) of such clusters are in $A \beta$ immunoreactive plaques (Figure 2B). Single swollen neurites were rarely seen and were never associated with plaques. Double staining for PHF1 and TS (Figure 2, C and D) revealed that 96 to $99 \%$ of PHF1positive clusters of swollen neurites are within TS-positive plaques and that 82 to $100 \%$ of TS-stained plaques contain such clusters. Therefore, virtually all clusters of phosphorylated tau-positive swollen neurites are found in $\mathrm{fA} \beta$ containing plaques.

\section{Neuronal Loss Is Found in the Immediate Vicinity of $f A \beta$-Containing Rhesus Plaques}

Scholl's concentric circles analysis (with five consecutive rings between concentric circles analyzed) demonstrated substantial loss of Nissl stained neurons in the immediate vicinity of compact $A \beta$ deposits in the aged rhesus cortex (Figure $3 A$ ). Overall, a significant $48 \%$ reduction in the number of neurons per $\mathrm{mm}^{2}$ was observed

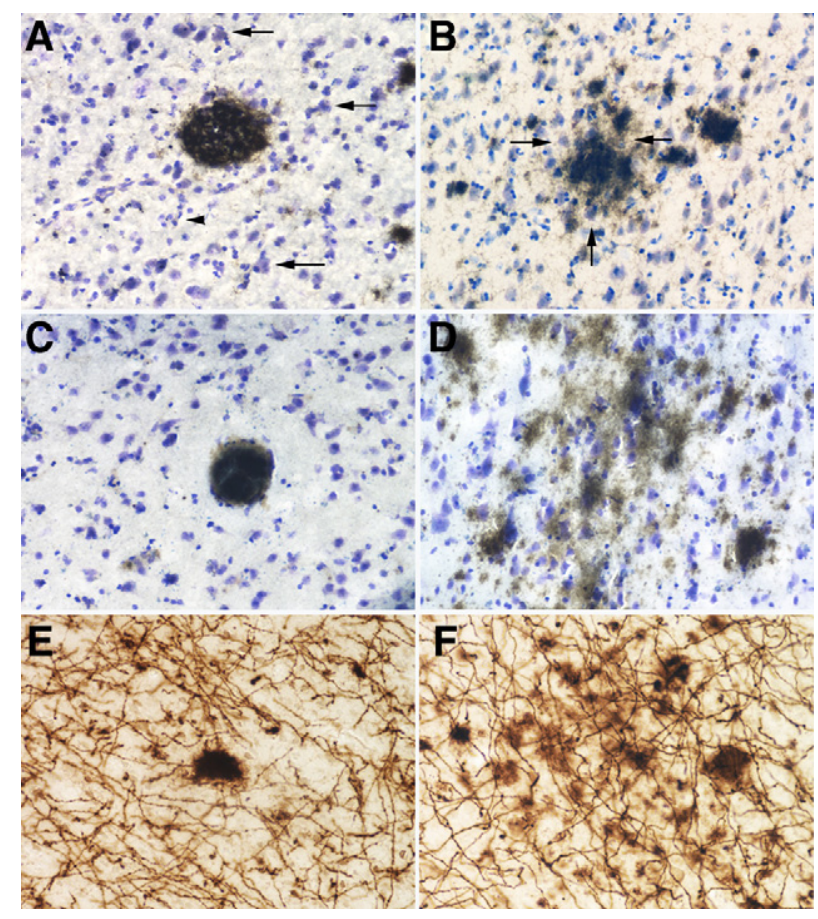

Figure 3. Neuronal and axonal loss is associated with compact plaques in the aged rhesus cortex. A: Compact $\mathrm{A} \beta$ immunoreactive plaques in the rhesus cortex were associated with significantly reduced numbers of Niss stained neurons (arrows) in their vicinity. In contrast, the area around such plaques displayed increased density of glial cells (arrowhead). B: No visible reduction in the numbers of neurons was seen in the vicinity of diffuse plaques. Intact neurons were observed in the vicinity and within such plaques (arrows). C: Compact plaques in the human brain displayed even greater loss of neurons in their vicinity when compared with the rhesus. D: Similar to the rhesus, diffuse plaques in the human cortex were not associated with loss of neurons in their vicinity. E: The area around compact plaques in the aged rhesus cortex displayed significant loss of acetylcholinesterase-positive cholinergic axons. F: Cholinergic axons were intact in the area surrounding diffuse $\mathrm{A} \beta$ immunoreactive plaques. Magnification in $\mathbf{A}-\mathbf{F}, \times 200$. 
A

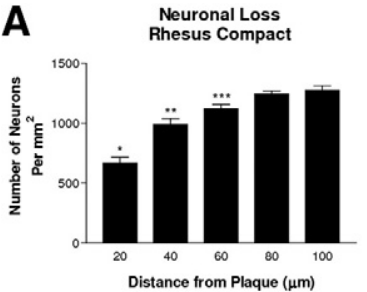

C

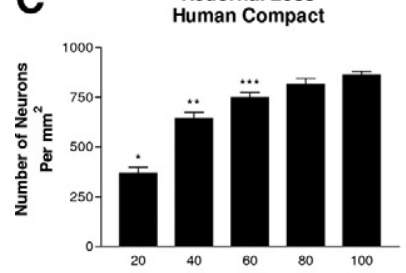

Distance From Plaque $(\mu \mathrm{m})$

E

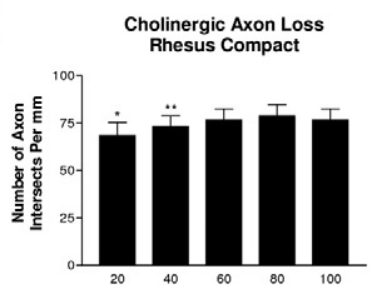

Distance from Plaque $(\mu \mathrm{m})$
B Neuronal Loss

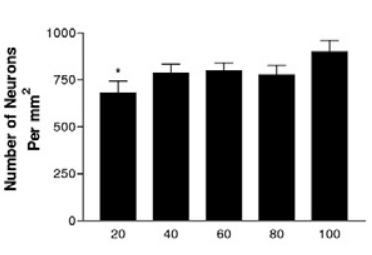

D

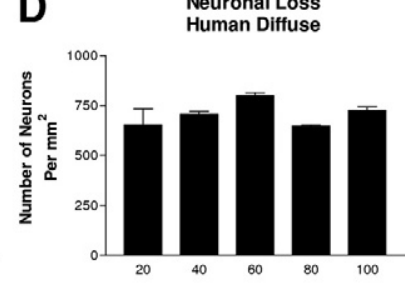

$\mathbf{F}$

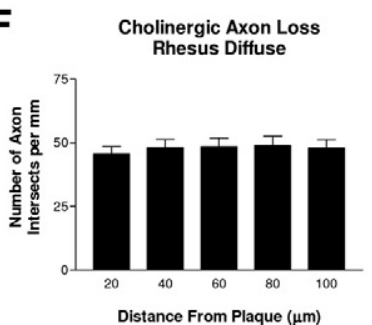

Figure 4. Results of Scholl's analysis revealed neuronal and cholinergic axonal loss in the vicinity of compact plaques that were progressively less pronounced with distance away from plaques. A: The ring bound by two circles $20 \mu \mathrm{m}$ apart in the immediate vicinity of compact plaques in the aged rhesus cortex contained significantly smaller numbers of neurons than the next four rings in the direction away form compact plaques $\left({ }^{*} P<0.001\right)$. The same was true of the second ring $\left({ }^{* *} P<0.001\right)$ and the third $\operatorname{ring}\left({ }^{* * * *} P<0.01\right)$ when compared with the rings away from plaques. There was no significant difference between the numbers of neurons in the fourth and fifth ring $(P>$ 0.05). B: In the area next to diffuse plaques, only the numbers of neurons in the first ring showed a small but statistically significant lower number of neurons when compared with the fifth ring $\left({ }^{*} P<0.01\right)$. No significant differences were found in the number of neurons among other rings. C: Similar but more pronounced loss of neurons was observed around compact plaques in the aged human and AD cortex. The numbers of neurons in the first ring next of compact plaques in the human cortex was significantly smaller than the number in the next four rings away from plaques ( ${ }^{*} P<$ 0.001 ), and this trend was progressively less pronounced with distance away from plaques $\left({ }^{* * *} P<0.001\right.$ when compared with rings $3-5$; ${ }^{* * * *} P<0.001$ when compared with rings 4 and 5; $P>0.05$ when comparing ring 4 and 5). D: In contrast to compact plaques, the numbers of neurons in five rings around diffuse plaques were not significantly different from each other $(P>0.05)$. E: The numbers of cholinergic axon intersects with the first circle around compact plaques in the aged rhesus cortex were significantly lower than intersects with the next four circles away from plaques $\left({ }^{*} P<0.001\right)$. The number of intersects with the second circle was significantly lower when compared with circle $4\left({ }^{* *} P<0.001\right)$. There were no other significant differences between numbers of intersects with other circles. F: There were no significant differences between numbers of axon intersects with any of five circles around diffuse plaques in the aged rhesus cortex $(P>0.05)$.

in a $20-\mu$ m-diameter ring around compact plaques when compared with the fifth consecutive ring away from plaques (Figure 4A). The number of neurons in the first ring was also significantly smaller when compared with those of the other three adjacent 20- $\mu$ m-diameter rings. The number of neurons per $\mathrm{mm}^{2}$ within the next ring was significantly less than those in the three subsequent rings. The number of neurons in the third ring was significantly smaller than the fourth and fifth rings, and those in the fourth and fifth rings were not significantly different from each other. Thus, the area around compact plaques

\section{Neuronal Loss \\ Rhesus Microglia}

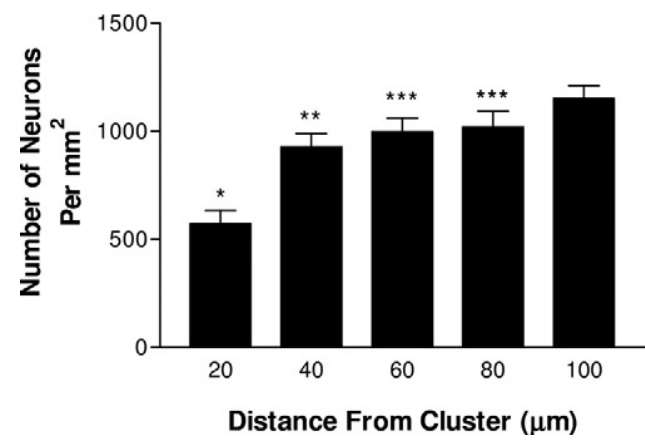

Figure 5. Neuronal loss is prominent in the vicinity of microglia clusters in the aged rhesus cortex, virtually all of which are in compact thioflavin-Spositive plaques that contain $\mathrm{fA} \beta$. The number of neurons in the first ring around microglia clusters was significantly smaller that the next four rings away from clusters $\left({ }^{*} P<0.001\right)$, and this trend became progressively less pronounced in subsequent rings around microglia clusters $\left({ }^{* * *} P<0.001\right.$ when compared with the fifth ring; ${ }^{* * *} P<0.01$ when compared with the fifth ring).

displays significant loss of neurons, which is progressively less pronounced when moving away from the plaque.

Next, we investigated the selectivity of neuronal loss in the vicinity of compact plaques (Figure 3B). The first $20-\mu \mathrm{m}$-diameter ring around diffuse plaques showed a small $13 \%$ loss of neurons, which was statistically significant only when compared with the numbers of neurons in the fifth ring. There were no significant differences in the numbers of neurons per $\mathrm{mm}^{2}$ among the other rings (Figure 4B).

To ensure that the results obtained using compact plaques chosen morphologically are the same as that for $\mathrm{fA} \beta$ containing plaques, we also quantified the number of neurons around activated microglia clusters, up to $98 \%$ of which are found in $\mathrm{fA} \beta$-containing TS-positive plaques. Using Scholl's analysis, we found identical loss of neurons around microglia clusters, which was progressively reduced as distance away from the clusters was traversed (Figure 5). These findings indicate that neuronal loss is selectively associated with compact plaques that contain detectable $\mathrm{fA} \beta$ within them.

\section{Neuronal Loss Is Selectively Associated with fA $\beta$ in Human Aged and AD Plaques}

To determine the relevance of the findings in aged rhesus to the process in the aged human and AD brains, a similar Scholl's analysis was performed in the vicinity of plaques in the human brain. The data obtained from normal aged and AD cases were nearly identical. Therefore the counts obtained for cases in the two groups were statistically analyzed together. A significant $57 \%$ loss of neurons was observed in the first $20-\mu \mathrm{m}$-diameter ring around compact plaques when compared with the other four rings away from such plaques (Figures $3 \mathrm{C}$ and $4 \mathrm{C}$ ). Similar to the aged rhesus brain, the loss of neurons became progressively less pronounced with distance away from the plaque, such that no significant difference was observed in the number of neurons between the 
fourth and the fifth rings. Thus, loss of neurons next to compact plaques in the human brain is similar to and more pronounced than that in the aged rhesus.

As is the case in the aged rhesus, plaque-associated loss of neurons in the human brain is selective to the compact variety. We found no significant differences in the numbers of neurons per $\mathrm{mm}^{2}$ among any of the five consecutive rings around diffuse plaques in the human brain (Figures 3D and 4D).

\section{Selective Loss of Cholinergic Axons Is Associated with Compact Plaques in Aged Rhesus}

Next, we investigated the status of AChE-positive cholinergic axons in the vicinity of plaques in the aged rhesus cortex. A Scholl's concentric circles analysis was performed in which the numbers of cholinergic axons intersecting five circles $20 \mu \mathrm{m}$ apart around plaques were used as data. A small but statistically significant decrease in the number of intersects of cholinergic axons with the first circle around compact plaques was observed when compared with intersects of the other four circles (10\% on average, Figures $3 E$ and $4 E$ ). The number of intersects with the second circle was significantly different from those with the fourth circle (7\% decrease). There was no significant difference between the numbers of intersects of other circles. Thus, modest loss of cholinergic axons is observed in the vicinity of compact plaques, which becomes progressively less pronounced with distance away from the plaque.

No significant differences were seen between the numbers of intersects of cholinergic fibers with five consecutive circles around diffuse plaques (Figures 3F and 4F). Therefore, the loss of cholinergic axons is specific to the area surrounding compact plaques.

\section{Discussion}

The results of the present set of experiments clearly demonstrate pathology selectively present in the vicinity of $f A \beta$-containing compact plaques in the aged rhesus, aged human, and $A D$ brains. $f A \beta$ containing compact plaques in the aged rhesus contained activated microglia and abnormally phosphorylated tau (PHF1)-positive swollen/dystrophic neurites. To our knowledge, this is the first demonstration of phosphorylated tau in swollen neurites associated with plaques in nonhuman primates. These findings clearly show that plaques in nonhuman primates possess the same characteristics as those in the human brain. ${ }^{9,11,13,14}$ Recent observations using in vivo multiphoton imaging have demonstrated microglia activation and appearance of dystrophic neurites associated with $\mathrm{f} A \beta$-positive plaques in animal models of AD. ${ }^{45}$

At present it is not clear whether the presence of plaque-associated dystrophic neurites found in $A D$ brains is related to the formation of neurofibrillary tangles, the main constituent of which is also abnormally phos- phorylated tau. We did not observe any tangles in the brains of the aged rhesus used in these experiments, nor has the presence of tangles in the rhesus brain been reported by others. Our observations suggest that either the process of dystrophic neurite and tangle formation are independent of each other or the former are the first to appear in the cascade of AD-type pathology. We did find morphologically normal neurons positive for the PHF1 epitope of phosphorylated tau, reminiscent of the pretangles observed in the aged human and AD brains. ${ }^{46}$ It remains to be shown whether such pretangles are related to the process of dystrophic neurite formation.

Significantly, we observed substantial loss of neurons in the immediate vicinity of plaques containing $f A \beta$ in the aged rhesus and human cortex. Such neuronal loss diminished progressively with distance away from plaques. Loss of neurons was virtually absent in the vicinity of diffuse plaques that do not contain $\mathrm{f} A \beta$. Thus, loss of neurons is a specific property of compact plaques that contain $\mathrm{f} A \beta$. These observations indicate that the loss of neurons observed within the area occupied by plaques ${ }^{35}$ also extends beyond the plaque to its immediate vicinity.

We also observed small but selective loss of cholinergic axons in the immediate vicinity of compact plaques. This observation is consistent with the general disruption of the neuropil attributed to plaques. ${ }^{34}$ The basal forebrain cholinergic system, including its cortically projecting axons, is subject to early pathology and damage in the course of normal aging and in AD. ${ }^{36}$ Our observations suggest that $\mathrm{f} A \beta$ in plaques may contribute, at least in part, to abnormalities in cortical cholinergic axons in AD.

Collectively, the findings of the present experiments demonstrate that the major pathologies observed in the brains of $A D$ patients-neuronal loss, axonal loss, activation of microglia, and abnormal phosphorylation of tau-are associated with $\mathrm{fA} \beta$ within plaques. In particular, our demonstration of neuronal and axonal loss in the vicinity of compact plaques containing $\mathrm{f} A \beta$ is strongly suggestive of the toxic effects of this $A \beta$ conformation on neurons. These observations indicate that the pathology we had observed after injections of $\mathrm{f} A \beta$ in the aged primate cortex ${ }^{21}$ is very likely a property of $\mathrm{f} A \beta$ in situ. We have recently demonstrated that inhibition of microglia activation significantly reduces loss of neurons after injections of $\mathrm{fA} \beta$ in the cerebral cortex of aged rhesus monkeys. ${ }^{47}$ Therefore, it is possible that the loss of neurons and axons observed in association with $\mathrm{fA} \beta$ containing plaques is mediated, at least in part, by activation of microglia.

\section{$A \beta$ Conformation and Neurotoxicity}

$A \beta$ exists in a number of conformations. The best studied of these are monomers, soluble oligomers, insoluble oligomers, protofibrils, and fibrils. Monomers are thought to be unstable and to form oligomers rapidly in solution, particularly when their concentration is high. Oligomers of a wide size range are soluble and eventually form insoluble aggregates deposited in diffuse plaques. Protofibrils are soluble precursors of mature $A \beta$ fibrils, the latter 
being insoluble. Because of their soluble characteristics and their ability to move freely within the neuropil, small oligomers and protofibrils can interfere with normal neuronal membrane function and have been demonstrated to hamper synaptic function ${ }^{22,23}$ and to inhibit fast axonal transport. $^{24}$ They are also associated with memory deficits in animal models of AD. ${ }^{48}$ These findings have led to the conclusion that soluble $A \beta$ species may make a significant contribution to the earliest manifestations of cognitive deficits and dementia in man.

Nearly all $A \beta$ conformations have been implicated in neuronal degeneration. A great number of in vitro studies indicate that $f A \beta$ is toxic to neurons. Exposure of neuronal cultures to $\mathrm{fA} \beta$ has been shown to cause significant neuronal loss. ${ }^{2,18,49,50}$ In vivo studies in the rodent have confirmed the toxic effects of $\mathrm{fA} \beta .^{18,19,51}$ Furthermore, our work demonstrated that the toxic effects of $\mathrm{f} A \beta$ are enhanced in the aged nonhuman primate brain. ${ }^{21}$ Small concentrations (200 pg) of $\mathrm{f} A \beta$, but not freshly prepared soluble $A \beta$, resulted in significant neuronal loss, activation of glial cells, and phosphorylation of tau when injected into the cerebral cortex of aged rhesus and marmoset monkeys. Thus, both in vitro and in vivo evidence indicate that $\mathrm{f} A \beta$ exerts toxic effects on neurons.

In vitro studies have also demonstrated that administration of soluble $A \beta$ oligomers and protofibrils cause degeneration of neurons in culture. ${ }^{5,22,25,28,52}$ Soluble $A \beta$ oligomers exert toxic effects on cultured cells in the absence of protofibrils or fibrils. ${ }^{25,53}$ However, unlike $\mathrm{f} A \beta$, the effects of extracellular $A \beta$ oligomers and protofibrils on neuronal death have only been demonstrated in vitro. Thus, the determination of whether these conformations of $A \beta$ can actually result in neuronal death in vivo must await future experiments. The fact that most transgenic mouse models of Alzheimer's disease, in which levels of soluble $A \beta$ oligomers and protofibrils are expected to be high early in life, show no or little loss of neurons ${ }^{54}$ argues against neuronal degeneration caused by these conformations in vivo. However, in at least some of these models, soluble $A \beta$ oligomers have been found to reduce synaptic proteins, ${ }^{55}$ a finding consistent with the postulated synato-toxicity of this conformation of $A \beta$. Of great interest, addition of the Arctic APP mutation, which is known to increase $A \beta$ fibrilogenesis in vitro, to an APP transgenic mouse line that displays learning and memory deficits, resulted in increased $A \beta$ aggregation and neuritic plaque formation, decreased soluble $A \beta$ oligomers, and improved learning and memory. ${ }^{48}$ Based on this and other observations, it is likely that aggregation of $A \beta$ in plaques and perhaps also $A \beta$ fibrilogenesis may be beneficial by immobilizing soluble $\mathrm{OA} \beta$ and preventing its synaptotoxicity. However, the evidence reviewed earlier suggests that over the long term, $f A \beta$ may cause neuronal death.

Intraneuronal accumulation of $A \beta$, most likely of the oligomeric conformation, has also been shown to exert toxic effects on neurons. Evidence indicates that $A \beta$ is generated intracellularly ${ }^{4}$ and accumulates in neurons in both $A D$ brain ${ }^{29,30}$ and in animal models of the disease. ${ }^{29,30,56,57}$ In a triple transgenic mouse model of $A D$, accumulation of $A \beta$ was shown to occur before plaque or tangle formation and to be correlated with deficits in learning and memory. ${ }^{57}$ Clearance of $A \beta$ via immunotherapy reversed the learning deficits. In the $5 \times$ transgenic mouse model of $A D$, in which levels of $A \beta$ are exceptionally high, significant accumulation of $A \beta$ in some cortical neurons precedes neuronal degeneration. ${ }^{56}$ However, it is not yet known whether this association is causative. Importantly, Takahashi et al 29,30 have clearly demonstrated that degenerative processes in synapses occur in neurons with accumulation of $A \beta$. These findings suggest that intraneuronal accumulation of $A \beta$ oligomers may be associated with degeneration similar to that observed following exposure to extracellular $\mathrm{fA} \beta$.

There is evidence that exchange and conversion occur among various conformations of $A \beta .^{58,59}$ Therefore, it could be argued that the neuronal loss observed in the vicinity of $\mathrm{f} A \beta$-containing compact plaques may be due to higher levels of soluble $A \beta$ oligomers and protofibrils surrounding plaques, resulting from constant exchange with $\mathrm{f} A \beta$, rather than to $\mathrm{f} A \beta$ itself. Soluble $A \beta$ oligomers are precursors to larger insoluble oligomers which are deposited in diffuse plaques. Therefore, it is likely that exchange among various conformations would cause high levels of soluble $A \beta$ oligomers in the area surrounding diffuse plaques. Yet we found no or very little loss of neurons and cholinergic axons around diffuse plaques, indicating that the loss of neurons we observed in compact plaques is unlikely to be due to soluble $A \beta$ oligomers. An exchange between conformations around $\mathrm{fA} \beta$ containing plaques would be expected to result in high concentrations of protofibrillar $A \beta$ in the vicinity of compact plaques. It is therefore conceivable that the loss of neurons and cholinergic axons we observed in association with compact plaques is influenced by protofibrillar $A \beta$ concentrations. However, no definitive effects can be attributed to $A \beta$ protofibrils in this regard until their in vivo toxicity is investigated and established.

\section{Functional Significance of Fibrillar A $\beta$ Pathology}

We observed clear loss of neurons and cholinergic axons in the vicinity of $\mathrm{fA} \beta$-containing compact plaques in the aged rhesus and human cortex. Loss of cortical neurons and axons is likely to interfere with cortical function and cognitive abilities. In the aged rhesus and human cortex, the relative abundance of $\mathrm{fA} \beta$ containing plaques is rather low. Thus, it is expected that the neuronal and axonal loss associated with these plaques cause either a small or no interference with cognitive function, giving rise to "normal" cognition in the nondemented elderly. However, it should be noted that cognitive decline in the elderly is an established phenomenon. This fact is reflected in age-appropriate adjustments in norms for neuropsychological tests. Thus, neuropsychological scores considered "normal" in an 80-year-old are considerably lower than those of a normal 50-year-old. Furthermore, a subpopulation of the elderly display mild cognitive impairment $(\mathrm{MCl})$ - considered a prodromal stage for dementia, particularly Alzheimer's disease-without being demented. ${ }^{60,61}$ It remains to be determined whether 
damage associated with compact plaques contributes to the above declines in cognitive function in the nondemented elderly.

In $A D$, compact plaques containing $f A \beta$ are found in relative abundance. In fact, the frequency of cortical compact plaques containing dystrophic neurites is used for the pathological diagnosis of AD. ${ }^{15}$ It is highly likely that the neuronal and axonal loss associated with $f A \beta$ in these plaques makes a major contribution to the cognitive abnormalities observed in AD. This conclusion is consistent with the findings of a strong correlation between the density of compact neuritic plaques and severity of dementia in this disorder. ${ }^{16,17}$

\section{References}

1. Selkoe DJ: Toward a comprehensive theory for Alzheimer's disease. Hypothesis: Alzheimer's disease is caused by the cerebral accumulation and cytotoxicity of amyloid beta-protein. Ann NY Acad Sci 2000, 924:17-25

2. Lorenzo A, Yankner BA: B-Amyloid neurotoxicity requires fibril formation and is inhibited by Congo red. Proc Natl Acad Sci U S A: 1994 , 91:12243-12247

3. Pike CJ, Walencewicz AJ, Glabe CG, Cotman CW: In vitro aging of beta-amyloid protein causes peptide aggregation and neurotoxicity. Brain Res 1991, 563:311-314

4. Walsh DM, Tseng BP, Rydel RE, Podlisny MB, Selkoe DJ: The oligomerization of amyloid beta-protein begins intracellularly in cells derived from human brain. Biochem 2000, 39:18031-18039

5. Hartley DM, Walsh DM, Ye CP, Diehl T, Vasquez S, Vassilev PM, Teplow DB, Selkoe DJ: Protofibrillar intermediates of amyloid betaprotein induce acute electrophysiological changes and progressive neurotoxicity in cortical neurons. J Neurosci 1999, 19:8876-8884

6. Kayed R, Head E, Thompson JL, Mclntire TM, Milton SC, Cotman CW, Glabe CG: Common structure of soluble amyloid oligomers implies common mechanism of pathogenesis. Science 2003, 300:486-489

7. Mann DMA, Brown AMT, Prinja D, Jones D, Davies CA: A morphological analysis of senile plaques in the brains of non-demented persons of different ages using silver, immunocytochemical and lectin histochemical staining techniques. Neuropathol Appl Neurobiol 1990, 16:17-25

8. Wisniewski HM, Bancher C, Barcikowska M, Wen GY, Currie J: Spectrum of morphological appearance of amyloid deposits in Alzheimer's disease. Acta Neuropathol 1989, 78:337-347

9. Terry RD, Wisniewski HM. Ultrastructure of senile dementia and of experimental analogues. In: Gaitz CM, editor. Aging and the Brain. New York: Plenum Press; 1972, 89-116

10. Sheng JG, Mrak RE, Griffin WS: Glial-neuronal interactions in Alzheimer disease: progressive association of IL-1alpha+ microglia and S100beta+ astrocytes with neurofibrillary tangle stages. J Neuropathol Exp Neurol 1997, 56:285-290

11. Fukumoto $H$, Asami-Odaka A, Suzuki N, Iwatsubo T: Association of $A$ beta 40-positive senile plaques with microglial cells in the brains of patients with Alzheimer's disease and in non-demented aged individuals. Neurodegen 1996, 5:13-17

12. Sheng JG, Mrak RE, Griffin WS: Neuritic plaque evolution in Alzheimer's disease is accompanied by transition of activated microglia from primed to enlarged to phagocytic forms. Acta Neuropathol 1997, $94: 1-5$

13. Perlmutter LS, Barron E, Chui HC: Morphologic association between microglia and senile plaque amyloid in Alzheimer's disease. Neurosci Lett 1990, 119:32-36

14. Trojanowski JQ, Shin RW, Schmidt ML, Lee VM: Relationship between plaques, tangles, and dystrophic processes in Alzheimer's disease. Neurobiol Aging 1995, 16:335-345

15. Mirra SS, Heyman A, McKeel D, Sumi SM, Crain BJ, Brownlee LM, Vogel FS, Hughes JP, van Belle G, Berg L: The Consortium to Establish a Registry for Alzheimer's Disease (CERAD). Part II: Standardization of the neuropathologic assessment of Alzheimer's disease. Neurology 1991, 41:479-486
16. Mena R, Wischik CM, Novak M, Milstein C, Cuello C: A progressive deposition of paired helical filaments (PHF) in the brain characterizes the evolution of dementia in Alzheimer's disease. J Neuropathol Exp Neurol 1991, 50:474-490

17. Nagy Z, Esiri MM, Jobst KA, Morris JH, King EM, McDonald B, Litchfield S, Smith A, Barnetson L, Smith AD: Relative roles of plaques and tangles in the dementia of Alzheimer's disease: correlations using three sets of neuropathological criteria. Dementia 1995, 6:21-31

18. Yankner BA: Mechanisms of neuronal degeneration in Alzheimer's disease. Neuron 1996, 16:921-932

19. Emre M, Geula C, Ransil BJ, Mesulam MM: The acute neurotoxicity and effects upon cholinergic axons of intracerebrally injected betaamyloid in the rat brain. Neurobiol Aging 1992, 13:553-559

20. Yankner BA, Duffy LK, Kirschner DA: Neurotrophic and neurotoxic effects of amyloid beta protein: reversal by tachykinin neuropeptides. Science 1990, 250:279-282

21. Geula C, Wu C-K, Saroff D, Lorenzo A, Yuan M, Yankner BA: Aging renders the brain vulnerable to amyloid $\beta$-protein neurotoxicity. Nat Med 1998, 4:827-831

22. Walsh DM, Klyubin I, Fadeeva JV, Cullen WK, Anwyl R, Wolfe MS Rowan MJ, Selkoe DJ: Naturally secreted oligomers of amyloid beta protein potently inhibit hippocampal long-term potentiation in vivo. Nature 2002, 416:535-539

23. Selkoe DJ: Alzheimer's disease is a synaptic failure. Science 2002, 298:789-791

24. Pigino G, Morfini G, Atagi Y, Deshpande A, Yu C, Jungbauer L, LaDu $\mathrm{M}$, Busciglio J, Brady S: Disruption of fast axonal transport is a pathogenic mechanism for intraneuronal amyloid beta. Proc Natl Acad Sci USA: 2009, 106:5907-5912

25. Chromy BA, Nowak RJ, Lambert MP, Viola KL, Chang L, Velasco PT Jones BW, Fernandez SJ, Lacor PN, Horowitz P, Finch CE, Krafft GA, Klein WL: Self-assembly of Abeta(1-42) into globular neurotoxins. Biochem 2003, 42:12749-12760

26. Demuro A, Mina E, Kayed R, Milton SC, Parker I, Glabe CG: Calcium dysregulation and membrane disruption as a ubiquitous neurotoxic mechanism of soluble amyloid oligomers. J Biol Chem 2005, 280:17294-17300

27. Kayed R, Sokolov Y, Edmonds B, Mclntire TM, Milton SC, Hall JE, Glabe CG: Permeabilization of lipid bilayers is a common conformation-dependent activity of soluble amyloid oligomers in protein misfolding diseases. J Biol Chem 2004, 279:46363-46366

28. Lambert MP, Viola KL, Chromy BA, Chang L, Morgan TE, Yu J, Venton DL, Krafft GA, Finch CE, Klein WL: Vaccination with soluble Abeta oligomers generates toxicity-neutralizing antibodies. J Neurochem 2001, 79:595-605

29. Takahashi RH, Milner TA, Li F, Nam EE, Edgar MA, Yamaguchi $H$, Beal MF, Xu H, Greengard P, Gouras GK: Intraneuronal Alzheimer abeta42 accumulates in multivesicular bodies and is associated with synaptic pathology. Am J Pathol 2002, 161:1869-1879

30. Takahashi RH, Almeida CG, Kearney PF, Yu F, Lin MT, Milner TA, Gouras GK: Oligomerization of Alzheimer's beta-amyloid within processes and synapses of cultured neurons and brain. J Neurosci 2004, 24:3592-3599

31. Salehi A, Bakker JM, Mulder M, Swaab DF: Limited effect of neuritic plaques on neuronal density in the hippocampal CA1 area of Alzheimer patients. Alzh Dis Assoc Dis 1998, 12:77-82

32. Knowles RB, Gomez-Isla T, Hyman BT: Abeta associated neuropil changes: correlation with neuronal loss and dementia. J Neuropathol Exp Neurol 1998, 57:1122-1130

33. Sheng JG, Zhou XQ, Mrak RE, Griffin WS: Progressive neuronal injury associated with amyloid plaque formation in Alzheimer disease. J Neuropathol Exp Neurol 1998, 57:714-717

34. Benes FM, Farol PA, Majocha RE, Marotta CA, Bird ED: Evidence for axonal loss in regions occupied by senile plaques in Alzheimer cortex. Neurosci 1991, 42:651-660

35. Urbanc B, Cruz L, Le R, Sanders J, Ashe KH, Duff K, Stanley HE, Irizarry MC, Hyman BT: Neurotoxic effects of thioflavin S-positive amyloid deposits in transgenic mice and Alzheimer's disease. Proc Natl Acad Sci USA: 2002, 99:13990-13995

36. Geula C, Nagykery N, Nicholas A, Wu CK: Cholinergic neuronal and axonal abnormalities are present early in aging and in Alzheimer disease. J Neuropathol Exp Neurol 2008, 67:309-318

37. Geula C, Mesulam M-M, Saroff DM, Wu C-K: Relationship between 
plaques, tangles, and loss of cortical cholinergic fibers in Alzheimer's disease. J Neuropathol Exp Neurol 1998, 57:63-75

38. Geula C, Mesulam M-M: Systematic regional variations in the loss of cortical cholinergic fibers in Alzheimer's disease. Cerebral Cort 1996 6:165-177

39. Mesulam M-M, Geula C: Overlap between acetylcholinesterase-rich and choline acetyltransferase-positive (cholinergic) axons in human cerebral cortex. Brain Res 1992, 577:112-120

40. Hanker JS, Thornburg LP, Yates PE, Moore HG: The demonstration of cholinesterases by the formation of osmium blacks at sites of Hatchett's brown. Histochemie 1973, 37:223-242

41. Tago H, Kimura H, Maeda T: Visualization of detailed acetylcholinesterase fiber and neuron staining in rat brain by a sensitive histochemical staining. J Histochem Cytochem 1986, 34:1431-1438

42. Geula C, Mesulam M-M: Special properties of cholinesterases in the cerebral cortex of Alzheimer's disease. Brain Res 1989, 498:185-189

43. Levey Al, Bolam JP, Rye DB, Hallanger AE, Demuth RM, Mesulam M-M, Wainer $\mathrm{BH}$ : A light and electron microscopic procedure for sequential double antigen localization using diaminobenzidine and benzidine dihydrochloride. J Histochem Cytochem 1986, 34:1449-1457

44. Sani S, Klink A, Traul D, Niaraki N, Wu C-K, Geula C: Distribution, progression and chemical composition of cortical amyloid-beta deposits in aged rhesus monkeys: similarities to the human. Acta Neuropathol 2003, 105:145-156

45. Meyer-Luehmann M, Spires-Jones TL, Prada C, Garcia-Alloza M, de Calignon A, Rozkalne A, Koenigsknecht-Talboo J, Holtzman DM, Bacskai BJ, Hyman BT: Rapid appearance and local toxicity of amyloid-beta plaques in a mouse model of Alzheimer's disease. Nature 2008, 451:720-724

46. Lauckner J, Frey P, Geula C: Comparative distribution of tau phosphorylated at Ser262 in pre-tangles and tangles. Neurobiol Aging 2003, 24:767-776

47. Leung E, Guo L, Bu J, Maloof M, El Khoury J, Geula C: Microglia activation mediates fibrillar amyloid- $\beta$ toxicity in the aged primate cortex. Neurobiol Aging 2009, doi: 10.1016.j.neurobiolaging.2009.02.025

48. Cheng IH, Scearce-Levie K, Legleiter J, Palop JJ, Gerstein H, Bien-Ly N, Puolivali J, Lesne S, Ashe KH, Muchowski PJ, Mucke L: Accelerating amyloid-beta fibrillization reduces oligomer levels and functional deficits in Alzheimer disease mouse models. J Biol Chem 2007, 282:23818-23828

49. Busciglio J, Lorenzo A, Yeh J, Yankner BA: beta-Amyloid fibrils induce tau phosphorylation and loss of microtubule binding. Neuron $1995,14: 879-888$

50. Mattson MP, Tomaselli KJ, Rydel RE: Calcium-destabilizing and neu- rodegenerative effects of aggregated beta-amyloid peptide are attenuated by basic FGF. Brain Res 1993, 621:35-49

51. Kowall NW, Beal MF, Busciglio J, Duffy LK, Yankner BA: An in vivo model for the neurodegenerative effects of beta amyloid and protection by substance P. Proc Nat Acad Sci U S A 1991, 88:7247-7251

52. Lambert MP, Barlow AK, Chromy BA, Edwards C, Freed R, Liosatos M, Morgan TE, Rozovsky I, Trommer B, Viola KL, Wals P, Zhang C, Finch CE, Krafft GA, Klein WL: Diffusible, nonfibrillar ligands derived from Abeta1-42 are potent central nervous system neurotoxins. Proc Natl Acad Sci U S A 1998, 95:6448-6453

53. Leissring MA, Farris W, Chang AY, Walsh DM, Wu X, Sun X, Frosch MP, Selkoe DJ: Enhanced proteolysis of beta-amyloid in APP transgenic mice prevents plaque formation, secondary pathology, and premature death. Neuron 2003, 40:1087-1093

54. Takeuchi A, Irizarry MC, Duff K, Saido TC, Hsiao AK, Hasegawa M, Mann DM, Hyman BT, Iwatsubo T: Age-related amyloid beta deposition in transgenic mice overexpressing both Alzheimer mutant presenilin 1 and amyloid beta precursor protein Swedish mutant is not associated with global neuronal loss. Am J Pathol 2000, 157:331-339

55. Mucke L, Masliah E, Yu GQ, Mallory M, Rockenstein EM, Tatsuno G Hu K, Kholodenko D, Johnson-Wood K, Mcconlogue L: High-level neuronal expression of abeta 1-42 in wild-type human amyloid protein precursor transgenic mice: synaptotoxicity without plaque formation. J Neurosci 2000, 20:4050-4058

56. Oakley H, Cole SL, Logan S, Maus E, Shao P, Craft J, GuillozetBongaarts A, Ohno M, Disterhoft J, Van Eldik L, Berry R, Vassar R: Intraneuronal beta-amyloid aggregates, neurodegeneration, and neuron loss in transgenic mice with five familial Alzheimer's disease mutations: potential factors in amyloid plaque formation. J Neurosci 2006, 26:10129-10140

57. Billings LM, Oddo S, Green KN, McGaugh JL, LaFerla FM: Intraneuronal Abeta causes the onset of early Alzheimer's disease-related cognitive deficits in transgenic mice. Neuron 2005, 45:675-688

58. Nichols MR, Moss MA, Reed DK, Cratic-McDaniel S, Hoh JH, Rosenberry TL: Amyloid-beta protofibrils differ from amyloid-beta aggregates induced in dilute hexafluoroisopropanol in stability and morphology. J Biol Chem 2005, 280:2471-2480

59. Narayanan S, Reif B: Characterization of chemical exchange between soluble and aggregated states of beta-amyloid by solution-state NMR upon variation of salt conditions. Biochem 2005, 44:1444-1452

60. Petersen RC: Mild cognitive impairment as a diagnostic entity. J Intern Med 2004, 256:183-194

61. Petersen RC, Smith GE, Waring SC, Ivnik RJ, Tangalos EG, Kokmen E: Mild cognitive impairment: clinical characterization and outcome. Arch Neurol 1999, 56:303-308 